\title{
Heartbeat: ECG approaches to early detection of atrial fibrillation
}

Atrial fibrillation (AF) is a major cause of morbidity and mortality with an increasing prevalence in our ageing population. Yet, we have few effective approaches to identifying patients at risk of AF so that many patients are diagnosed only when they present with a stroke or heart failure. In this issue of Heart, Sanz-Garcia and colleagues $^{1}$ present a simple ECG-based score that predicts subsequent AF with a predictive value area under the curve (AUC) of 0.776 (95\% CI: 0.738 to 0.814 ). This computer assisted ECG approach was developed and validated in a cohort of 16316 patients with the risk score based on age (up to four points), $\mathrm{P}$ wave duration in lead aVF (>200 ms, 2 points) and lead V4 (>150 ms, 0.7 point), $\mathrm{T}$ wave duration in lead V3 $>250 \mathrm{~ms}$ (0.4 point), mean QTc interval $>450 \mathrm{~ms}$ ( 0.3 point), transverse $\mathrm{P}$ wave clockwise rotation $(0.3$ point) and frontal QRS complex clockwise rotation (0.4 point) (figure 1 ).

In the accompanying editorial, Kashou and Noseworthy ${ }^{2}$ point out that, compared with clinical AF risk scores, this ECG approach relies on one source of data (eg, the ECG) and thus may be easier to implement in clinical practice. Compared with artificial intelligence ECG models in which the specific ECG variables are unknown, the model proposed here uses identifiable, easily measured ECG variables (figure 2). Whatever approach is taken, accurate risk models offer the hope of routine screening for AF risk, facilitating early treatment and prevention of complications. As Kashou and Noseworthy conclude "Further study, including randomised controlled trials, is needed to refine the proposed AF risk-prediction models and to evaluate their application to patient care, optimising clinical workflow and alleviating the burden of this only growing healthcare crisis."

Point-of-care (or hand-held) cardiac ultrasound is increasingly available and offers the potential for early diagnosis of left ventricular (LV) dysfunction in patients presenting with shortness of breath. In a meta-analysis of 33 studies with 6062 participants,

Division of Cardiology, University of Washington, Seattle, Washington, USA

Correspondence to Professor Catherine M Otto, Division of Cardiology, University of Washington, Seattle, WA 98195, USA; cmotto@uw.edu

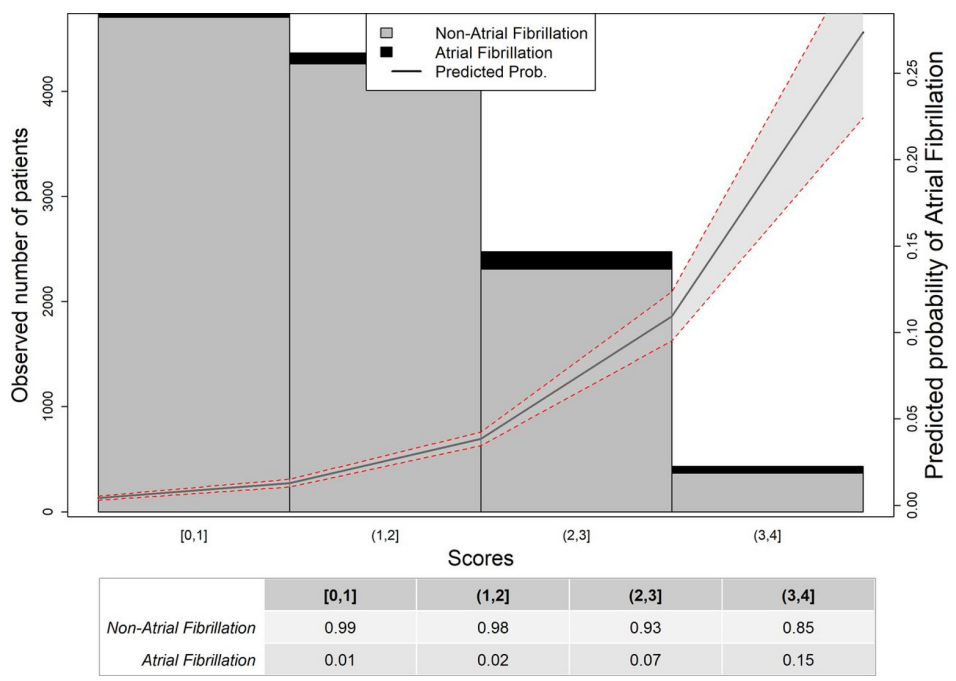

Figure 1 Probability of AF based on risk score values. Bars show the number of patients in the training cohort for each score value (non-AF in grey and AF in black). The trend line shows the estimated probability of AF. The table below represents the percentage of patients in the training cohort for each score value. $A F$, atrial fibrillation.

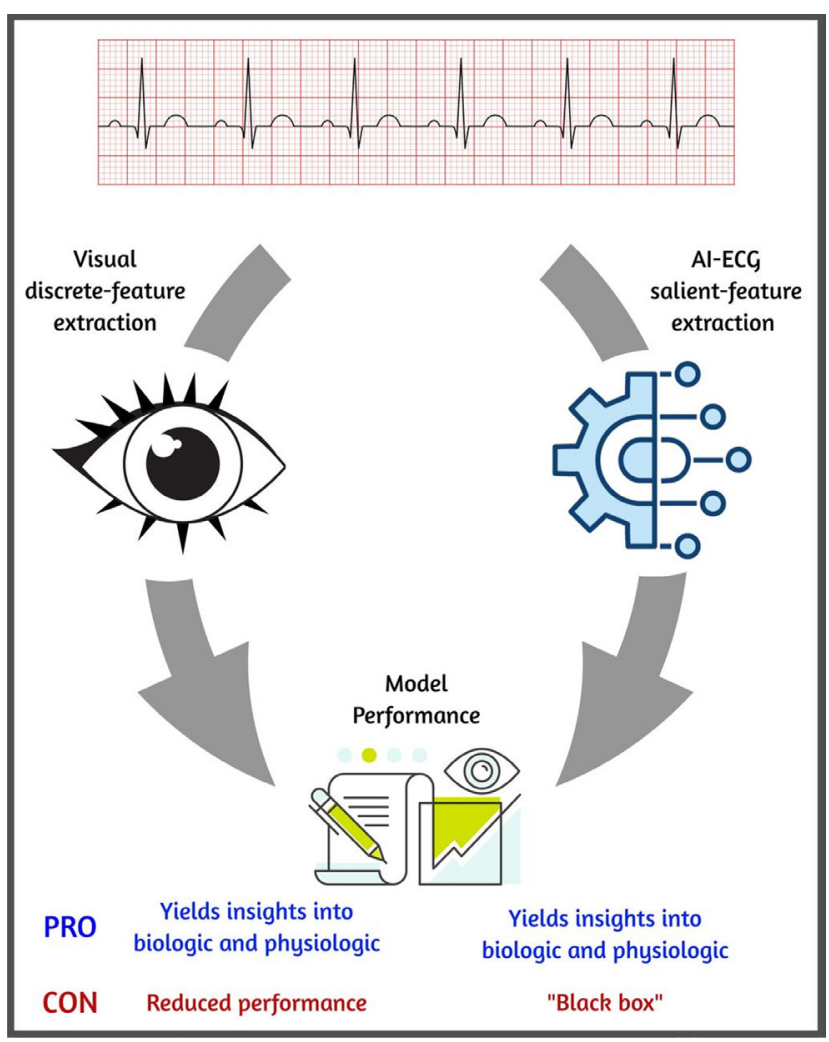

Figure 2 Pros and cons of visual discrete-feature extraction and AI-ECG salient-feature extraction. 


\begin{tabular}{|c|c|c|c|c|}
\hline Review question & \multicolumn{4}{|c|}{$\begin{array}{l}\text { Is handheld echocardiography able to accurately diagnose LV dysfunction compared } \\
\text { with TTE? }\end{array}$} \\
\hline Population & \multicolumn{4}{|c|}{$\begin{array}{l}6062 \text { participants aged } 65 \pm 5 \text { years with a male predominance of } 54 \% \text { requiring } \\
\text { routine referral for TTE }\end{array}$} \\
\hline Setting & \multicolumn{4}{|c|}{ Single centres with access to TTE } \\
\hline Studies & \multicolumn{4}{|c|}{ Studies of diagnostic tests } \\
\hline Quality of evidence & \multicolumn{4}{|c|}{$\begin{array}{l}\text { Majority of studies reported consecutive or random sampling, blinding of assessors } \\
\text { and short time between HUD and TTE imaging (24-28 hours) }\end{array}$} \\
\hline \multirow{2}{*}{ Pooled results } & \multicolumn{2}{|c|}{ Sensitivity $(95 \% \mathrm{Cl})$} & \multicolumn{2}{|c|}{ Specificity $(95 \% \mathrm{Cl})$} \\
\hline & Experienced & Inexperienced & Experienced & Inexperienced \\
\hline LVEF (any abnormality) & 88 (81 to 92$)$ & 83 (71 to 90$)$ & 96 (90 to 98$)$ & 89 (81 to 93$)$ \\
\hline LVEF (moderate/severe) & 93 (89 to 96$)$ & 84 (72 to 92$)$ & 96 (87 to 99$)$ & 91 (83 to 95$)$ \\
\hline WMA & 85 (76 to 91$)$ & 78 (70 to 84 ) & 95 (93 to 96$)$ & $88(85$ to 90$)$ \\
\hline LV dilatation & 89 (64 to 97) & 68 (51 to 81$)$ & 98 (93 to 99$)$ & 95 (93 to 96$)$ \\
\hline LVH & 85 (72 to 92$)$ & 80 (61 to 91$)$ & 91 (82 to 96$)$ & 87 (67 to 96$)$ \\
\hline
\end{tabular}

HUD, handheld ultrasound devices; LV, left ventricular; LVEF, LV ejection fraction; LVH, LV dilatation and hypertrophy; TTE, transthoracic echocardiography; WMA, wall motion abnormalities.

Jenkins and colleagues found that handheld-ultrasound imaging by an experienced operator, compared with a complete diagnostic echocardiogram, had a sensitivity of $88 \%$ and specificity of $96 \%$ for diagnosis of a reduced LV ejection fraction, with similar findings for diagnosis of a regional wall motion abnormality, LV dilation and LV hypertrophy. ${ }^{3}$ However, with inexperienced operators sensitivity was lower, although specificity remained high (table 1 ).

Nascimento and Beaton point out in the accompanying editorial ${ }^{4}$ that there is no question that hand-held ultrasound devices 'have advanced to the point, where they can be used to reliably evaluate LV function in different scenarios'; instead the issue now is providing adequate training and ensuring competency, as illustrated by the differences seen between experienced and inexperienced operators. The current challenge is to develop reliable methods for taskshifting that incorporate rigorous, yet efficient training protocols that enable scale-up of these technologies.' As they conclude: 'More than ever, robust accuracy data as presented in this meta-analysis, aligned with
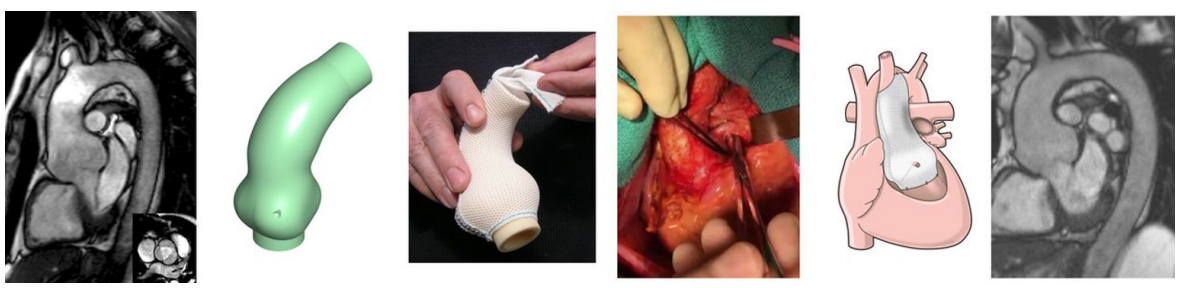

Figure 3 Ilustration of the PEARS concept. A preoperative CT or MRI scan is used to create a model of the patient's aorta, which is 3D-printed. A sleeve of polyethylene terephthalate mesh is shaped on this former. The resulting ExoVasc is implanted around the patient's aorta, from the ventriculo-aortic junction to the brachiocephalic artery. Postoperative imaging shows stable aortic dimensions and patent coronary orifices at 16 years postoperatively in the first patient. Figure reproduced with permission. ${ }^{9}$ care for patients with Marfan syndrome is valve-sparing root replacement (VSSR), with resection of the dilated sinuses and ascending aorta followed by placement of a surgical graft, reimplantation of the patient's native aortic valve inside the graft, and attachment of the coronary ostia to the graft. When preservation of the native valve is not possible, a composite prosthetic valve and aortic graft is needed. They point out that a technical challenge with the PEARS procedure is in situ isolation of the coronary arteries, which is more difficult than ostial reimplantation. In addition, 'the ability to properly anchor the graft at the level of the aortic annulus/ basal ring is imperative in preventing future annular dilation and resultant aortic regurgitation. The accuracy of suture placement on a beating, intact aortic root, especially in proximity to the right coronary cusp, may prove difficult and lead to late failures of the PEARS procedure.' Although randomised controlled clinical trials comparing different approaches to aortic surgery for inherited aortopathies are unlikely, longer term outcome data in larger patient groups are needed for the PEARS procedure.

The Education in Heart article ${ }^{7}$ in this issue reviews the assessment and management of peripheral vascular disease. Many patients with heart disease also have (or are at risk for) peripheral vascular disease so that an awareness of diagnostic and treatment approaches, as well as management of risk factors, is essential for all clinical cardiologists.

In a state-of-the-art review article, Debono and colleagues ${ }^{8}$ provide an update on endovascular repair for abdominal aortic aneurysms, another disease condition that often coexists with heart disease. The article focuses on endoleaks, the presence of blood outside the endovascular repair but within the aneurysm, including the approach to periodic surveillance imaging and indications for re-intervention.

Funding The authors have not declared a specific grant for this research from any funding agency in the public, commercial or not-for-profit sectors.

\section{Competing interests None declared.}

Patient and public involvement Patients and/or the public were not involved in the design, or conduct, or reporting, or dissemination plans of this research.

Patient consent for publication Not applicable.

Provenance and peer review Commissioned; internally peer reviewed.

(c) Author(s) (or their employer(s)) 2021. No commercial re-use. See rights and permissions. Published by BMJ.

$$
\text { Check for updates }
$$

To cite Otto CM. Heart 2021;107:1765-1767.

Heart 2021;107:1765-1767. 
doi:10.1136/heartjnl-2021-320418

\section{ORCID iD}

Catherine M Otto http://orcid.org/0000-0002-05279392

\section{REFERENCES}

1 Sanz-García A, Cecconi A, Vera A, et al. Electrocardiographic biomarkers to predict atrial fibrillation in sinus rhythm electrocardiograms. Heart 2021;107:1813-9.
2 Kashou A, Noseworthy P. Predicting incident atrial fibrillation in sinus rhythm: more than just trusting the 'black box'. Heart 2021;107:1770-1.

3 Jenkins S, Alabed S, Swift A, et al. Diagnostic accuracy of handheld cardiac ultrasound device for assessment of left ventricular structure and function: systematic review and meta-analysis. Heart 2021;107:1826-34.

4 Ramos Nascimento B, Zawacki Beaton A. Improved standardisation of training needed to achieve the potential of handheld echocardiography. Heart 2021;107:1772-3.

5 Van Hoof L, Rega F, Golesworthy T, et al. Personalised external aortic root support for elective treatment of aortic root dilation in 200 patients. Heart 2021;107:1790-5.

6 Burke CR, Bavaria J. The personalized external aortic root support procedure: interesting niche or ready for prime time? Heart 2021;107:1768-9

7 Tran B. Assessment and management of peripheral arterial disease: what every cardiologist should know. Heart 2021;107:1835-43.

8 Debono S, Nash J, Tambyraja AL. Endovascular repair for abdominal aortic aneurysms. Heart 2021:107:1783-9.

9 Pepper J, Golesworthy T, Izgi C. Personalised external aortic root support (PEARS) tostabilise an aortic root aneurysm. Br J Cardiol 2020;27:87-92. 